\section{PACE for speed}

\section{By Kai-Jye Lou, Staff Writer}

Researchers at Harvard University have developed an in vivo platform for the directed evolution of proteins and other macromolecules that could work faster and require less labor than traditional methods of directed evolution. ${ }^{1}$ The researchers are using their method, dubbed PACE (phage-assisted continuous evolution), to discover improved variants of existing proteins and macromolecules that could be used to manipulate genes or modify gene products.

Directed evolution is a process for engineering proteins that works by introducing selective pressures that favor mutations conferring desired properties. Each round of evolution has a mutation, selection and amplification step and usually takes a few days due to the time required to complete each step.

Multiple protein discovery platforms, such as the MolecularBreeding and CodeEvolver platforms at Maxygen Inc. and Codexis Inc., respectively, carry out directed evolution and use a method called DNA shuffling for the mutation step.

In DNA shuffling, related genes are digested into fragments and then recombined to form a new library of chimeric genes. For the selection step, assays are used to screen and select library members with the activity desired by the user.

These newly selected library members are amplified and sent through a subsequent round of digestion and recombination if an acceptable candidate has not been found.

To speed things up, the Harvard team decided to automate many of the existing steps by using a bacteriophage to introduce a gene of interest into Escherichia coli host cells. Bacteriophage is a viral vector with two properties that could make it useful for continuous evolution. First, the phage has a fast life cycle. Second, DNA sequences introduced by the phage are prone to mutations, and these are passed on to the virus' progeny.

By leaving the steps of the evolutionary process up to the phage and the host cell it infects, intervention from a human or robot is no longer needed at each step in continuous evolution. The end result is a system in which each round of evolution takes minutes instead of days.

"We chose to use bacteriophage because it introduces genes into cells as part of its life cycle and because it supports the mutation of the genes that it introduces," which meet some of the key requirements for evolution, said David Liu, professor of chemistry and chemical biology at Harvard and an investigator at the Howard Hughes Medical Institute.
In industry, the bacteriophage is recognized for its utility in phage display, which is used to identify molecules that bind with high affinity to target ligands. However, the utility of phage in directed evolution and for the discovery of molecules with high levels of enzymatic activity is not well established.

As test cases, the researchers showed that their PACE method could execute dozens of rounds of evolution in a single day, did not require user intervention between each round and evolved phage that encoded genes for mutant proteins that had up to several hundredfold increased enzymatic activities over the corresponding wild-type proteins (see Figure 1, "Schematic of phage-assisted continuous evolution").

Results were published in Nature.

"PACE enables researchers to evolve proteins with activities of interest more quickly than current methods and is also less labor intensive," said Liu. "The PACE method could aid the discovery of new proteins and macromolecules-including those with therapeutically relevant activities - that are not easily designed on a reasonable time scale using conventional directed evolution approaches."

"With conventional approaches, for each round of evolution, you will need to manually extract your material from the system, do a round of mutagenesis and then reinsert your material into a new set of cells," said James Collins, professor of biomedical engineering at Boston University and investigator at HHMI. "This process is fairly laborious, and a single round of evolution will usually take days, and in some cases, weeks."

"In the case of some desired evolutionary goals, such as enzymes with highly changed activities or specificities, the number of rounds of evolution necessary to access desired molecules may be very large," said Liu.

Liu said PACE can perform up to 40 rounds of evolution in 24 hours.

"One additional goal we worked hard to achieve is to keep the infrastructure requirements for using the PACE system consistent with that which would be available to a standard molecular biology lab. We didn't want our approach to require custom equipment or robotics, which might be difficult for labs to access," he added.

"The primary advantage of this approach is its ease and its ability to continuously carry out evolution on a molecule, which could dramatically reduce the time it takes to obtain molecules with the activities you want," said Collins.

"While it is possible in principle to carry out 200 rounds of evolution using standard approaches, it would be an extremely laborious process. Most would get tired after going through 5-10 rounds of evolution using standard approaches," said Andreas Plückthun, a professor in the Department of Biochemistry at the University of Zurich and cofounder of the human antibody company MorphoSys AG and Molecular Partners AG, which develops nonimmunoglobulin proteins called DARPins (designed ankyrin repeat proteins) as alternatives to antibody-based therapeutics. 


\section{Proving worth to industry}

Plückthun said the PACE approach expands the repertoire of methods for evolving new molecules but is unlikely to replace all existing methods. "For example, I think the approach could be useful for the evolution of enzymes because it can select for molecules with higher levels of catalytic activity, and this currently has to be done step by step. But it would probably be easier to select for molecules with high-affinity binding using phage and especially ribosome display because it is difficult to create selection pressures in the cytoplasm that favor affinity."

Plückthun noted that in ribosome and phage display, selections are done in solution and not in the cytoplasm of a cell; thus, the conditions can be fine-tuned, and non-natural compounds and competitors can be used, if needed.

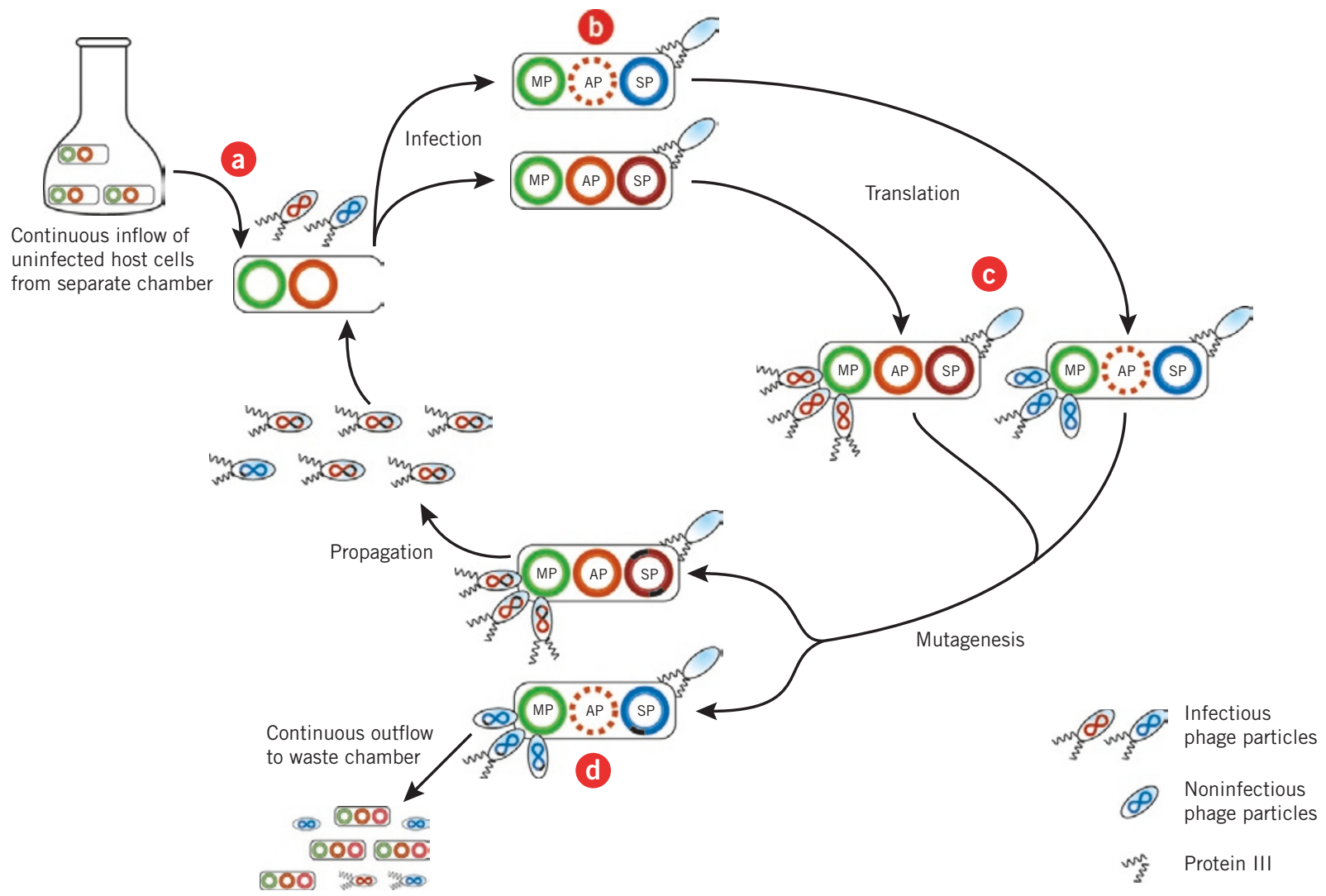

Figure 1. Schematic of phage-assisted continuous evolution. Phage require protein III to effectively infect host cells, and viral particles lacking this protein are about 100 million times less infectious than wild-type particles. ${ }^{2}$

Esevelt et al. exploit this property for a directed evolution approach they call phage-assisted continuous evolution (PACE). In this method, researchers link the production of protein III in the M13 filamentous bacteriophage to an activity of interest by deleting the associated gene from the DNA of the phage vector and inserting a copy of the gene into an accessory plasmid (orange circle; AP) in Escherichia coli host cells. The accessory plasmid is designed such that molecules with higher levels of the activity of interest increase protein III production. The $E$. coli host cells also carry a mutagenesis plasmid (green circle; MP) that researchers can use to modulate the error rate during DNA replication.

Host cells that flow into a chamber are infected with selection phage from a library [a]. Selection phage encoding genes for molecules that have higher levels of the activity of interest (red circle; SP) will induce greater expression of protein III production from the bacterial cell's accessory plasmid than phage encoding genes for less active or nonfunctional molecules (blue circle; SP) [b].

Infected cells that produce more protein III will generate a larger number of infectious phage progeny that also encode the gene for the more active molecule [c]. These phage will propagate in larger numbers and infect more cells than those encoding molecules with lower levels of activity.

While inside the host cell, phage DNA also can acquire mutations that affect the activity of the encoded molecule [d]. These mutations result in the generation of phage encoding gene variants that are not found in the original library. Researchers control the rate of mutagenesis via the mutagenesis plasmid.

The constant outflow from the system means that phage must propagate in sufficient numbers to maintain their presence. Thus the system continuously selects for phage encoding molecules with higher levels of the desired activity.

Figure adapted from Esevelt et al. ${ }^{1}$ 
Willem Stemmer, CEO of Amunix Inc. and cofounder of Maxygen, said the PACE approach is very innovative and would be of interest in the academic setting, but he had doubts about the method in a commercial setting.

"In directed evolution, it is very important that the candidate molecule is evolved under conditions that mimic the final setting in which it will be used. Otherwise, you probably won't get the desired final result," he told SciBX. "Standard phage display allows the selection of proteins for binding properties under conditions that can be controlled, but the approach described in the Liu paper only allows selection for properties that can be selected inside E. coli, which is highly limiting."

Moreover, Liu said that PACE currently can only evolve traits that can turn on gene expression. "If you want to evolve an enzyme that processes one metabolite into another, where neither metabolite causes significant changes in the level of gene expression, it would be difficult to use PACE to evolve such a molecule," he said.

However, he noted that the development of new methods to link a wider variety of molecular activities to gene expression could make PACE broadly applicable over time.

Christian Zahnd, CEO and cofounder of Molecular Partners, wanted to know how difficult it would be to control the selection pressure in the system and to steer the direction of evolution during PACE. "With conventional evolutionary approaches, the user is able to change and adjust the selection pressures during each round of evolution and can also bias the types of mutations that occur," he told SciBX.

"If the researchers could show that the method could be used to evolve an improved version of the best available version of commercially relevant molecules, it would be an important step towards establishing the utility of the approach for industry," Stemmer told SciBX.

"In the study they claim they could evolve molecules that promote protein-protein interactions, which could be one of the most interesting applications of this technology," said Zahnd. "It would be very interesting for them to demonstrate additional examples where they could use this approach to evolve molecules that are able to promote protein-protein interactions."

Liu and colleagues now are using PACE to discover variants of proteins with new or improved activities and to evolve a variety of macromolecules that can manipulate genes or modify gene products, including some that could have therapeutic relevance. His group also is using PACE to study basic questions about the nature of evolution that are impractical to answer using conventional laboratory evolution methods.

Harvard and HHMI have filed patent applications covering PACE. The IP is available for licensing through the university's Office of Technology Development.

Lou, K.-J. SciBX 4(18); doi:10.1038/scibx.2011.503

Published online May 5, 2011

\section{REFERENCES}

1. Esevelt, K.M. et al. Nature; published online April 10, 2011; doi:10.1038/nature09929

Contact: David R. Liu, Harvard University, Cambridge, Mass. e-mail: drliu@fas.harvard.edu

2. Nelson, F.K. et al. Virology 108, 338-350 (1981)

COMPANIES AND INSTITUTIONS MENTIONED

Amunix Inc., Mountain View, Calif.

Boston University, Boston, Mass.

Codexis Inc. (NASDAQ:CDXS), Redwood City, Calif.

Harvard University, Cambridge, Mass.

Howard Hughes Medical Institute, Chevy Chase, Md.

Maxygen Inc. (NASDAQ:MAXY), Redwood City, Calif.

Molecular Partners AG, Schlieren, Switzerland

MorphoSys AG (Xetra:MOR), Martinsried, Germany

University of Zurich, Zurich, Switzerland 